\title{
Transformed objects: the influence of unruly product design
}

\author{
Athanasios Manavis ${ }^{1}$, Georgios Pliatsios ${ }^{1}$,Eva Dimou ${ }^{1}$, Apostolos Korlos ${ }^{2}$, \\ and Panagiotis Kyratsis ${ }^{1, *}$ \\ ${ }^{1}$ Western Macedonia University of Applied Science, Department of Mechanical Engineering and \\ Industrial Design, Kila Kozani GR50100 Greece \\ 2 Alexander Technological Educational Institute of Thessaloniki, Department of Automotive \\ Engineering, P.O. Box 141, Thessaloniki, GR57400, Greece
}

\begin{abstract}
Nowadays, the image of a product becomes more important than the product itself. Unruly design is defined as the methodology of all objects that are designed with the intention to undermine the existing design-paradigm of the functionalists. At the same time, the term surreal object is based on the magical, transformative concept of the juxtaposition of diverse elements usually found in everyday objects. The present paper outlines the general principles of the unruly design and surrealism art movement, together with their influence on styles of the conceptual product design. A number of illustrative case studies are depicted, through an ergonomic approach, in order to ensure an appropriate interface relationship between art and product design. The products' transformations have been selected in order to stress the idea that using unruly design offers additional advantages in creating concepts from a different point of view. Innovation and unusual product design can create the basis for a complete product design transformation culture.
\end{abstract}

\section{Introduction}

Product design is about making things that can lead to change. Designers, through specific acts, bring into life not only solutions that help people to overcome problems, but also artefacts that offer opportunities for innovation in the field of aesthetics. More specifically, product aesthetics defines the object that gives pleasure and delight, through the incorporation of aesthetic features [1-3].

Nowadays, the image of the product becomes more important than the product itself. Furthermore, the relationship between beauty, usability and functionality shows the importance of aesthetics in product design (Fig. 1) [4]. A modern theory that describes the relationship between the image of the product and the product itself is the unruly design. The core of unruly design theory is based on both the postmodern and surrealism art movements (Fig. 2).

\footnotetext{
*Corresponding author: pkyratsis@teiwm.gr
} 


\section{The Three Factors}

\subsection{Unruly Design}

Unruly design is defined as the methodology of all objects that are designed with the intention to undermine the existing design-paradigm of the functionalists [5]. In this case, the definition of the product functionality is extended with emotional and cultural aspects, at the cost of usability, functionality and beauty. An unruly designed product follows five basic principles, the: a) combination of different interest domains, b) inspiration from popular culture, c) incorporation of a form complexity, d) use of ready-mades and e) use of uncommon materials [6].

The main influence of unruly design in product development is that, it can support designers to understand the implementation of meaning into demand driven design practice, and therefore extends the possibilities for making meaningful objects.

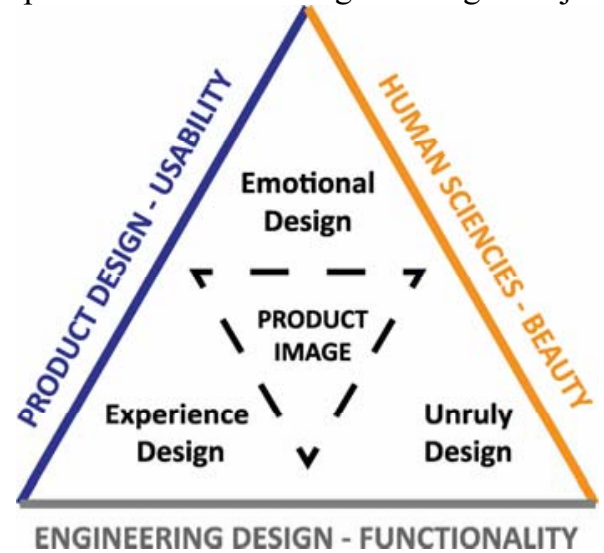

Fig. 1. The product image triangle.
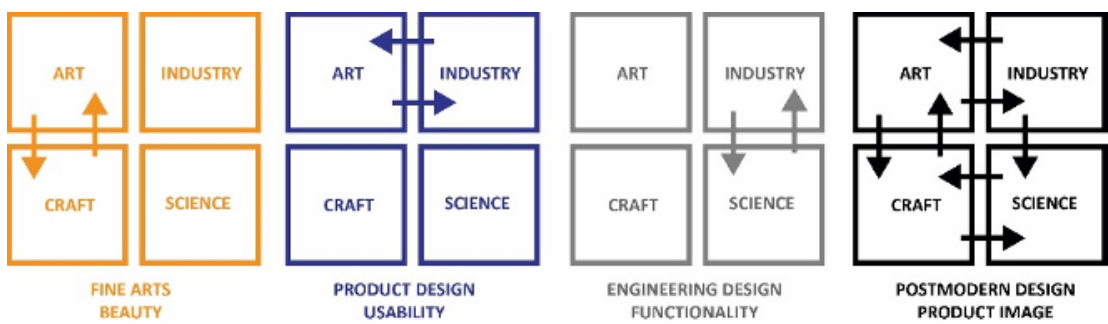

Fig. 2. Design theories and relationships between art, industry, craft and science fields [2].

\subsection{Surreal Object}

Surrealism occupies a unique place within the history of the $20^{\text {th }}$ century avant-garde because of its international influence and longevity. The Manifesto of Surrealism explains this art movement as a primarily tributary creation of an interior model, where dreams, the subconscious and automatism in creation, inspired a poetry designed to deny reality and throw it into turmoil [7-9]. The most well-known surrealist artist Salvador Dalí gave an initial definition to objects with a symbolic function. These objects led themselves to a minimum of mechanical functioning and they are based on the fantasies and representations that can arise from the performance of subconscious acts. 


\subsection{Ergonomics}

Ergonomics scopes to build a bridge between design parameters and human compatibility factors. It makes design more successful for user's acceptance. It is more than just product $\&$ user relationship tool. Design ergonomics is also an advancement in its journey, crossing the concept of the user, the product and function, the look at the user, while offers a product relationship in totality. A human user of a product is not a physical and cognitive processor. His needs are to be addressed in tune with his emotions, values, hopes, fears, and anxiety over new adaptations in life [10-12].

\section{Transformed Objects}

Transformed objects are a combination of all three factors unruly design, surreal object and ergonomics (Fig. 3). In this paper, several illustrative case studies are presented, through an ergonomic approach, in order to ensure an appropriate interface relationship between both the art and product design.

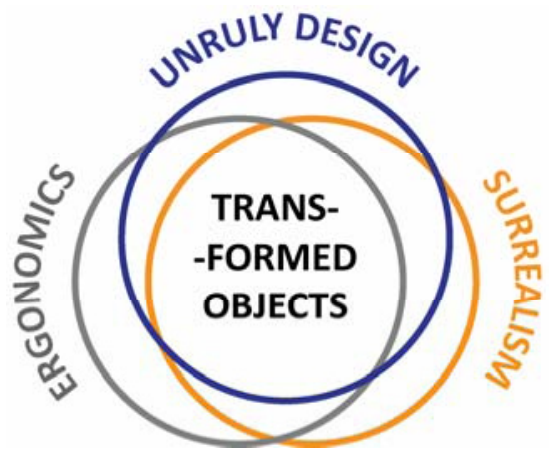

Fig. 3. The three factors of transformed objects.

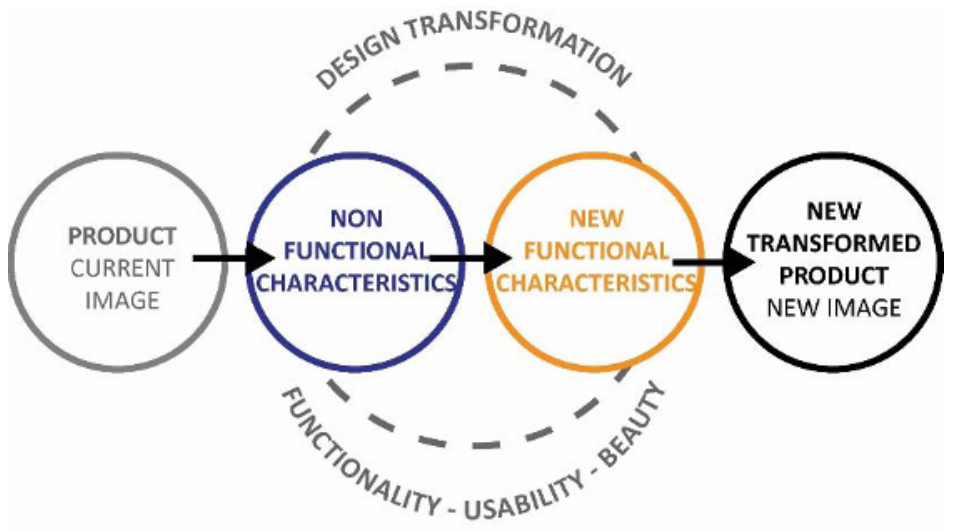

Fig. 4. The design process of transforming objects.

Figure 4 depicts the whole process used when the case studies were prepared. Initially, the designer chooses a full functional, useful and "beautiful" product. The next step is him/her to decide what product characteristics might be cancelled or manipulated from the function 
and the ergonomic point of view. The third stage relates with a conceptual product design, using design sketches for the development of the final idea of the novel transformed object. All these new criteria (functional, ergonomic and aesthetics) discovered at previous stages, are added at the new object. Finally, a transformed object is defined when following this creative design process. The aim is to build a new product image in addition to the current one. The whole framework of transformed objects offers opportunities to designers for developing creativity and experimentation opportunities. This provides an additional path for innovation and unusual product design that can offer a solid basis for a complete product design transformation culture.

\subsection{Wearable media player design}

The watch as a product is something personal. Everyone has its own taste and preferences in design and style. Watches are small timepieces intended to be carried or worn by a person. They are designed to keep working, despite the motions caused by personal activities. The five features that describe the ideal watch are: a) the material of the product (a titanium or ceramic watch is considered of higher value), b) the kind of watch glass used (a quality watch always has sapphire glass in addition to crystal glass), c) the design of the watch mechanism (a good, affordable watch is based on the quartz principle or uses a certified mechanical mechanism), d) its water resistance and e) the quality of its strap. All these features are selected with an aim to manipulate the users' perception from the conceptual design point of view (Fig. 5).

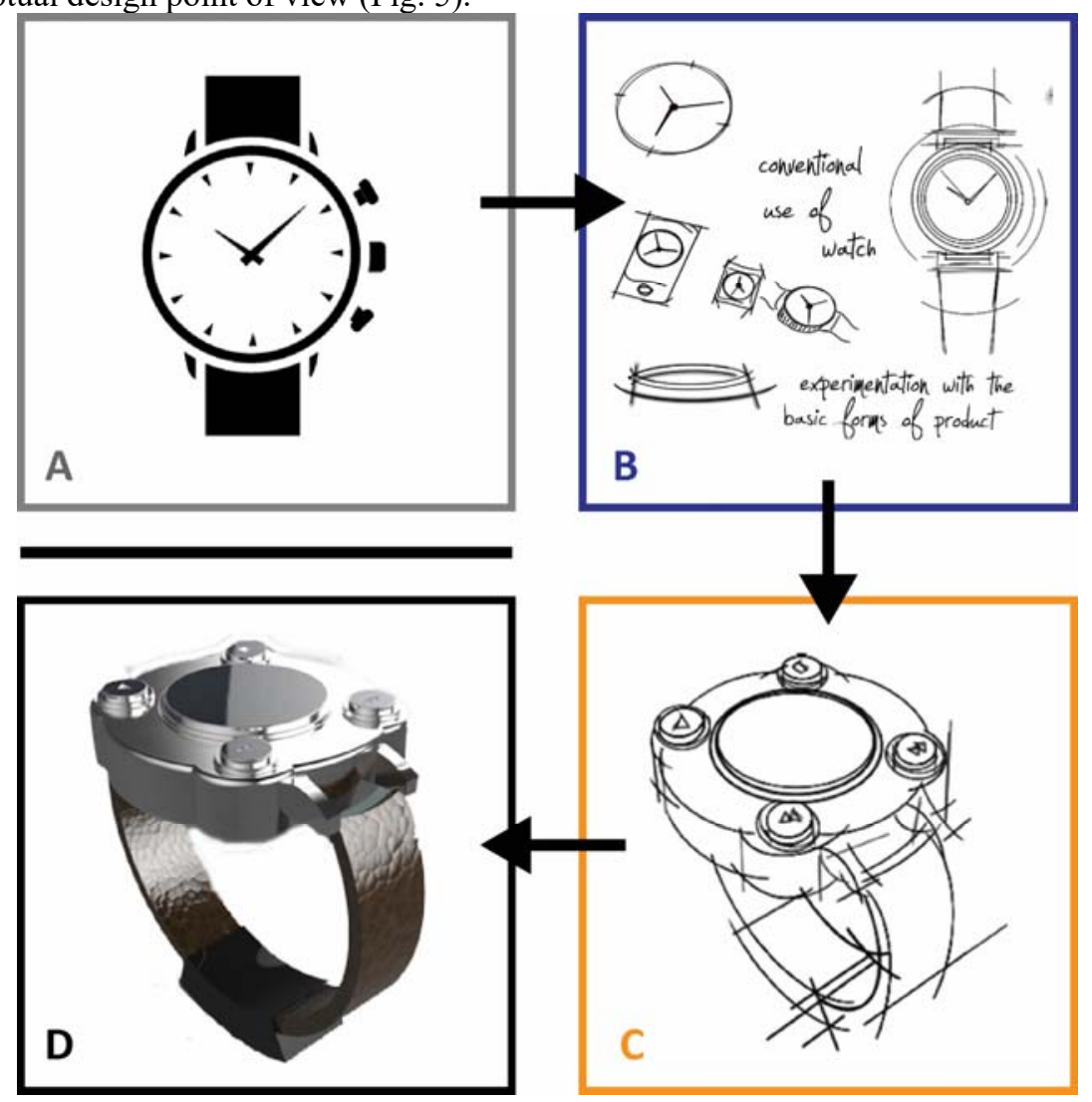

Fig. 5. A watch is transformed to a wearable media player: a) current image of the watch; b) nonfunctional characteristics; c) new functional characteristics; d) new transformed product. 
This procedure showed that a watch without ergonomics could be used i.e. with a cut strap, a blind screen and nonfunctional buttons. This specific design transformation guides to fundamental questions for the new product image, such as "what is the new role of this object?", "what features are to be replaced for the novel image of this product?"

At the third stage of the design process, the novel identity of a wearable media player with updated features is presented. The cut strap is replaced by a magnetic clip, the blind screen is substituted by a high-resolution monitor (like smartphones) and the nonfunctional buttons are redesigned to be fully ergonomic and functional keys. Finally, the main purpose of the initial watch is replaced from the final concept that promotes music. In addition, it underlines the difference between an anxiolytic watch and an entertainment media player.

\subsection{Data Storage Device design}

A key is a device that is used primary to operate a lock. A typical key is a small piece of metal consisting of four parts: a) tip, b) cuts, c) shoulder and d) bow. In this case, a typical key is transformed into a USB data storage device by the transformed object design process proposed (Figure 6). The experimentation with the basic forms of the product, guides the rotation of the door key upper part. Then an extrusion of the surface leads to a different geometry. During this step, the addition of the USB geometry should be highlighted.

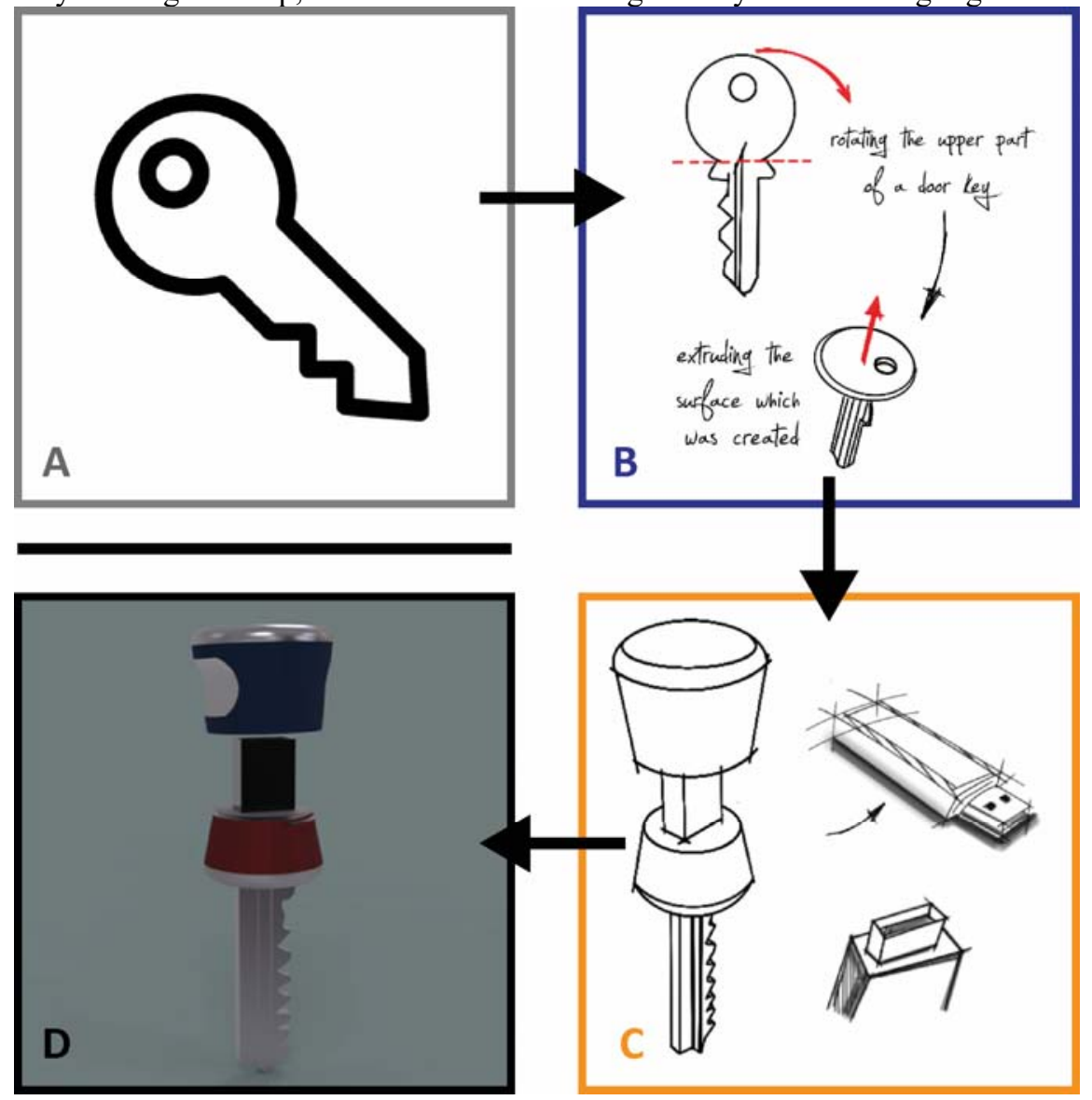

Fig. 6. A key is transformed to a USB stick. 
New functional characteristics are explored and the role of the cap leads to the data storage device geometry. Finally, a new image of this transformed product appears as a novel data storage device that is included in a fully functional, ergonomic and "beautiful" key. Two functions were incorporated in one ergonomic object. A unique approach of using all the three factors: unruly design, surrealism and ergonomics.

\section{Conclusions}

A new methodology was presented, based on the creation of transformed objects. The proposed framework could offer a series of great design and marketing advantages to enterprises that would be willing to follow this path. Transformed objects are a combination of three factors: unruly design, surrealism object and ergonomics. This specific approach makes use of shapes and functions that customers associate with a number of different original products and produces unusual items.

The relationship between beauty, usability and functionality shows the importance of aesthetics in product design. The proposed conceptual designs incorporate not only innovative creative design, but the creation of novel "product images". A new chain of products based on this unusual approach could trigger further new product designs and design methodologies.

\section{References}

1. P. Moutaftsi, P. Kyratsis J APPL PACKAGING RES, 8(3), 1 (2016).

2. P. Kyratsis, N. Efkolidis, Ath. Manavis, St. Peristeri, ACAD J MAN ENG, 11(4), 56, (2013)

3. P. Hekkert, H. Leder, Product Aesthetics: Product Experience (Elsevier Science, 2008)

4. M. Hassenzahl, Product Experience: Aesthetics in interactive products: correlates and consequences of beauty (Elsevier Science, 2008)

5. W. Eggink, A. Reinders, $5^{\text {th }}$ International Congress of International Association of Societies of Design Research, Tokyo (2013)

6. W. Eggink, $4^{\text {th }}$ World Conference on Design Research. The International Association of Societies of Design Research (IASDR) (2011)

7. A. Breton, Manifestoes of Surrealism (University of Michigan Press, 1969)

8. R. Spiteri, A Companion to Dada and Surrealism, 110 (2016)

9. E.H. King, A Companion to Dada and Surrealism, 416 (2016)

10. L. Rungtai, J.G. Kreifeldtb, INT J IND ERGONOM, 27, 259 (1999)

11. L. Rungtai, Proceedings of the Human Factors Society (1992)

12. L. Rungtai, J.G. Kreifeldt, Proceedings of the Human Factors Society (1992) 